\title{
STATISTICAL MODELING OF COVID-19 PANDEMIC STAGES WORLDWIDE
}

\author{
Ranadeep Datta ${ }^{1}$, Prabhat Kumar Trivedi ${ }^{1}$, Ayush Kumawat ${ }^{1}$, Rahul Kumar ${ }^{1}$, Isha Bhradwaj ${ }^{1}$, \\ Neha Kumari ${ }^{1}$ Varun Agiwal $^{1,2}$, Saurabh Kumar ${ }^{1,3}$, Ashok Kumar ${ }^{1,4}$ and Ashutosh Shukla ${ }^{5}$, \\ and Jitendra Kumar ${ }^{1, *}$ \\ ${ }^{1}$ Central University of Rajasthan, Bandarsindri, Kishangarh, Ajmer, Rajasthan, India \\ ${ }^{2}$ Department of Community Medicine, Jawaharlal Nehru Medical College, Ajmer, Rajasthan \\ ${ }^{3}$ Invertis University Bareilly, Uttar Pradesh, India \\ ${ }^{4}$ SHKM Government Medical College, Nuh, Hariyana \\ ${ }^{5}$ National Statistical Office, Field and Operationn Division, Agra, Uttar Pradesh, India
}

\begin{abstract}
COVID-19 is an infectious disease, growth of which depends upon the linked stages of the epidemic, the average number of people one person can infect and the time it takes for those people to become infectious themselves. We have studied the COVID-19 time series to understand the growth behaviour of COVID-19 cases series. A structural break occurs in the COVID-19 series at the change time form one stage to another. We have performed the structural break analysis of data available for 207 countries till April 20, 2020. There are 42 countries which have recorded five breaks in COVID cases series. This means that these countries are in the sixth stage of growth transmission and show a downward pattern in reporting in the daily cases, whereas countries with two and three breaks, record the rapid growth pattern in the daily cases. From this study, we conclude that the more the breaks in the series, there is more possibility to determine the constant or decreasing rate of daily cases. It is well fitted using lognormal distribution as this distribution is archived at its highest peak after some period and then suddenly it decreases at a longer time period. This can be seen in various countries like China, Australia, New Zealand and so on.
\end{abstract}

Keywords: COVID-19, Structural Break, Growth Stage

*Corresponding author at: Department of Statistics, Central University of Rajasthan, Bandersindri, Ajmer, India. Email addresses: vjitendrav@ gmail.com 


\section{INTRODUCTION:}

COVID-19 is a novel coronavirus that has travelled from Wuhan, China, to the most of the countries (207) within 100 days. COVID-19 is an infectious disease, growth of which depends upon the stage of the epidemic, the average number of people one person can infect and the time it takes for those people to become infectious themselves. However, the growth rate of coronavirus cases is different in every country depends upon its health infrastructure, persons living life, environmental conditions and many other factors. So, the stages of disease transmission depends upon the changing pattern in the series of the number of COVID cases based on total population, total land area, medical facilities, etc. These changing patterns may be analyzed by a structural break model where shifting in the series can be determined using a change in the growth of spreading of COVID-19. Hence, the structural break can easily explain the shifting on the COVID-19 time series from one breakpoint to another by changing the model parameters. For each break interval, it follows a well-known growth model, and the growth rate of the series is different. Significant contribution in the study of a structural break in time series includes the work of Chow (1960), Nelson and Plosser (1982), Andrews (1993), Bai and Perron (1998), Chaturvedi and Kumar (2007), Bai (2010), Meligkotsidou et al. (2011, 2017) and Agiwal et al. (2018).

In COVID-19 cases, a structural break might occur when most of the population and land area are affected, sudden increments in corona patients on daily basis, population not following the government guidelines or other factors. It also depends upon the administrative model which has the main goal to slow down the growth of COVID-19 cases by various measures like social distancing, lockdown etc. Health services may be provided in a more structural and significant way when such services try to increase the recovery of COVID-19 cases. Most of the countries have managed the disease in reasonable time. However, some countries record the change in the COVID series, i.e., there is a possibility in the series that structural break(s) have happened. So, the present study has analyzed the changing pattern of COVID-19 cases and identified various suitable breakpoints. For that, we have determined the breakpoints using statistical methodology and then examined the changing trend in each break interval. We have analyzed every stage separately to understand the behaviour of transmission on infections. Various distribution models have been fitted in each break interval based on the number of infected days and the number of total cases. Based on the results, we determine the best-fitted model in the overall break interval.

In the analysis of break intervals, the Lognormal distribution fitted better among all the five discrete and continuous distributions under consideration. Similarly, in the analysis of the number of cases at breaks, the Lognormal distribution fitted better than the rest of the distributions, both discrete and continuous, under consideration. 


\section{MATERIAL \& METHOD}

The data has been collected from our World COVID-19 databases of daily updates of confirmed cases. The data covers the total number of people infected with the COVID-19 virus from December 31, 2019, to April 20, 2020. As of April 20, 2020, there were 2350993 cases of infections worldwide. On April 20, there were a total of 205 countries/provinces infected with the virus. This data was processed for further analysis.

In the present paper, we apply the Chow F-test statistic to determine the potential breaks at all change points in the COVID series. This methodology is well discussed in R package "strucchange", developed by Zeileis et al. (2002). The number of change points $(\mathrm{m})$ is determined by breakpoint (s) function in strucchange that employs the location of the break(s) using the minimum value of the residual sum of squares (RSS) and Bayesian information criterion (BIC) whereas. Various discrete and continuous distributions are fitted for all break intervals to show the development of the COVID19 cases feasibly. The selection of the best-fitted distribution is based on the minimum value of the Akaike information criterion (AIC) and Bayesian information criterion (BIC).

We will do the following analysis step by step:

Step-1: Determine the number of the structural break(s) and its locations.

Step-2: Classify the countries by taking the break interval of World COVID cases.

Step-3: Fit a distribution in each break interval based on total infected days and the total number of cases.

Step-4: Display the histogram and density plot based on the results obtained from the previous steps to conclude the COVID-19 cases.

\section{RESULTS}

Out of the total 205 countries infected till April 20, 2020, 10 countries do not have any breaks in their data, 3 countries have only 1 break, 23 countries have 2 breaks, 48 countries have 3 breaks, 79 countries have 4 breaks and remaining 42 countries have five breaks. All breaks identification depends upon the size of the series as well as how much times there is a shift in the series. We can interpret the number of breaks as various growth stages of COVID-19 cases. First breakpoint shows the change in the growth stage of COVID-19 cases at the initial time period. Those countries having a single breakpoint can be considered in the first stage because in these countries the growth of corona cases is increasing slowly. A country can be regarded as in the second and third stages of growth in COVID cases where there is a rapid change in the trend of corona cases. These countries have recorded two or three breakpoints. Some of the countries that are having three breaks are getting a higher peak and record a decreasing trend in the growth of cases. During the analysis, some countries like Australia, Austria, China, Greece, New Zealand and many more record a constant or decreasing growth rate in the COVID-19 cases. These countries show the fourth and fifth stage of growth and have recorded at higher numbers of breaks (four, five) in the series. These breakpoints are identified 
based on the trend pattern of corona cases. So, we can consider break intervals as the growth stages of COVID cases in our analysis.

\section{Analysis the stages based on duration time:}

All infectious disease always depends majorly on two factors, the first number of carriers and the second time of infections. So, in the present section, we study the number of days in various COVID19 infection stages. First, the structural breaks are found for each country over the data of total cumulative cases. Maximum five breaks (B1, B2, B3, B4 and B5) are identified in each country series. Then intervals are formed between all the breakpoints, namely zero stage (S1: 1st day to B1), the first stage (S2: $(\mathrm{B} 2+1)$ to B3), second stage (S3: B2+1 to B3), the third stage (S4: B3+1 to B4), fourth stage (S5: B4+1 to B5) and fifth stage (S6: (B5+1 to April 20). Here, each country has different number of breaks, and its locations are also different. So, we study the total number of days where there is a change in the series that means infection growth is from one stage to another stage. This means that each break interval for every county records the time to shift from one to another point and it is termed as I-stage, II-Stage, III-Stage, IV-Stage, Stage-V and Stage-VI. Table 1 records the descriptive statistics of the occurrence period of different stages.

Table 1 shows that the shortest duration between any two breaks in the series is four days. The average number of days for any interval is approximately eight days, with an exception in the first interval, which is highest about 20 days. The quartiles for the different intervals show that $75 \%$ of the countries have 6 to 25 days before coming into the first stage of the outbreak.

The common dataset of the number of days in the break intervals seems to be positively skewed as recorded in above Figure 1. The later intervals are found to have a smaller number of days in break intervals as compared to the others. The individual break interval datasets have similar asymmetrical plot like the collective interval data, shown in Figure 2. The plots are skewed to the right, and there are fewer countries that have larger break intervals.

Similarly, for the rest of the intervals, most of the countries have about 4 to 9 days before the next breakpoint occurs. The difference in the shape of the first interval and rest of the intervals is that there are more countries in the S1 than the rest, i.e., almost all the countries have observed their first breakpoint based on total number of cases within the country. Likewise, very few countries have observed their sixth stage in the number from the total cases, which leads to a distorted histogram for S6 and less skewed than the other intervals. This also shows a control situation of these countries that observed the sixth stage of COVID-19 infection.

The Cullen and Frey graphs were fitted for each of the intervals and used to find possible candidates for fitting various discrete and continuous distributions to the data. The following distributions are considered: Normal, Negative Binomial, Poisson, Lognormal and Gamma distribution. Table- 2 describes the best-fitted distribution based on various assumed distribution. This table shows that lognormal distribution is fitted well in all break intervals except S4.

Tracing the theoretical density and distribution function over the empirical density and distribution are displayed in Figure 3 and 4. These figures give a nice view of the shape of each stage 
interval of COVID-19 infection and fits well. Points the on P-P plots lie on the straight line representing a better fit, whereas in Q-Q plot departures from the straight line can be observed which increase at the later stages (due to fewer data in later stages). Deviations from the straight line are a hint of lack of fit.

\section{Analysis the number of cases at each stage:}

In the analysis of different COVID-19 infection, we also need to understand the COVID-19 cases as the infection in equally crucial for the number of carriers. So far, we modelled COVID-19 cases in different stages of spreading of COVID-19. First, the data consisted of distinctively very high values contributed by countries with a high number of infected cases. These are the countries like the United States, Italy, China, Spain, Germany, Iran, etc., these data points consisted of about $15-19 \%$ of the data and has been removed considering outliers which are recorded in Table 3.

The outlier countries are the countries that are worse affected by the disease than the countries in the corresponding stages. It is also recorded that countries which are severely affected by COVID19 (United States, China, etc.), are not having reached in last stages like fifth and sixth. Table 4 records the descriptive statistics of the break interval based on total cases, and Figure 5 displays its density plot.

The aggregate data, after removing outliers, have a positively skewed shape and mildly peaked in the first half of the data. The average number of cases at B0 is 74 , which is reasonable as these are moderately affected (after removing outlier) countries and therefore have fewer cases till the first break. This average increased rapidly till B4, reaching approximately 580 cases, where about $75 \%$ of the countries have less than 802 cases in total. At break point B5, this average falls. The reason behind this is a possibility to decrease the growth pattern of the COVID-19 cases in these countries. Most of these countries have a high number of infected cases that were pulling the average up at the earlier breaks.

The suitable candidates for distribution fitting were found using Cullen and Frey graphs in Table 5. The lognormal distribution fitted the best among exponential, normal, Gamma and other discrete distributions. The trace of density \& distribution function and the P-P plots are shown in Figures 7-8. These figures show that a better fitting is observed using the lognormal distribution as it has achieved its peak and after that, there is a sudden decreasing trend in the extended time period. This can be easily seen in China series. Departures from the straight line in the Q-Q plot at the higher quantiles form the distribution is fitted due to the highly skewed countries data. Hence the methods other than lognormal distribution are not able to thoroughly explain the skewness of the data accurately in the total cases.

\section{DISCUSSION}

This paper studies and analyzes the various stages in the growth of COVID-19 cases using the structural break methodology. We considered the cumulative cases of coronavirus for each country as a data series and identified the breaking point when the structure of the series is shifted suddenly. We 
have examined the inference based on each break period. This break period provides the length of the duration and number of cases in various growth stages. For each break interval, we fit a distribution to explain the growth pattern of COVID-19 cases. Based on the results, we observed that lognormal distribution is better fitted to the number of days and number of cases in each break interval of COVID19 in the whole world. This is so because lognormal distribution has a long right tail with an exponential growth pattern.

\section{REFERENCES}

1. Andrews, D. W. (1993). Tests for parameter instability and structural change with unknown change point. Econometrica, 61(4):821-856.

2. Bai, J. and Perron, P. (1998). Estimating and testing linear models with multiple structural changes. Econometrica, 66(1):47-78.

3. Chaturvedi, A. and Kumar, J. (2007). Bayesian unit root test for time series models with structural breaks. American Journal of Mathematical and Management Sciences, 27(12):243-268.

4. Chow, G. C. (1960). Tests of equality between sets of coefficients in two linear regressions. Econometrica, 28:591-605.

5. Meligkotsidou, L., Tzavalis, E., and Vrontos, I. (2017). On Bayesian analysis and unit root testing for autoregressive models in the presence of multiple structural breaks. Econometrics and Statistics, 4:70-90.

6. Meligkotsidou, L., Tzavalis, E., and Vrontos, I. D. (2011). A Bayesian analysis of unit roots and structural breaks in the level, trend, and error variance of autoregressive models of economic series. Econometric Reviews, 30(2):208-249.

7. Nelson, C. R. and Plosser, C. R. (1982). Trends and random walks in macroeconomic time series: some evidence and implications. Journal of Monetary Economics, 10(2):139-162.

8. Bai, J. (2010). Common breaks in means and variances for panel data. Journal of Econometrics, 157(1):78-92.

9. Agiwal V., Kumar J. and Shangodoyin D. K. (2018): A Bayesian Inference of Multiple Structural Breaks in Mean and Error Variance in Panel AR(1) Model. Statistics in Transition, 19(1):7-23.

10. Singh, A. K., Singh, A. and Engelhardt, M. (1997): The lognormal distribution in environmental applications. In Technology Support Center Issue Paper.

11. Delignette-Muller, M. L. and Dutang, C. (2015): fitdistrplus: An R package for fitting distributions. Journal of Statistical Software, 64(4):1-34.

12. Stedinger, J. R. (1980): Fitting log normal distributions to hydrologic data. Water Resources Research, 16(3):481-490. 
13. Sánchez, S., Ancheyta, J., and McCaffrey, W. C. (2007): Comparison of probability distribution functions for fitting distillation curves of petroleum. Energy \& Fuels, 21(5): 2955-2963. 


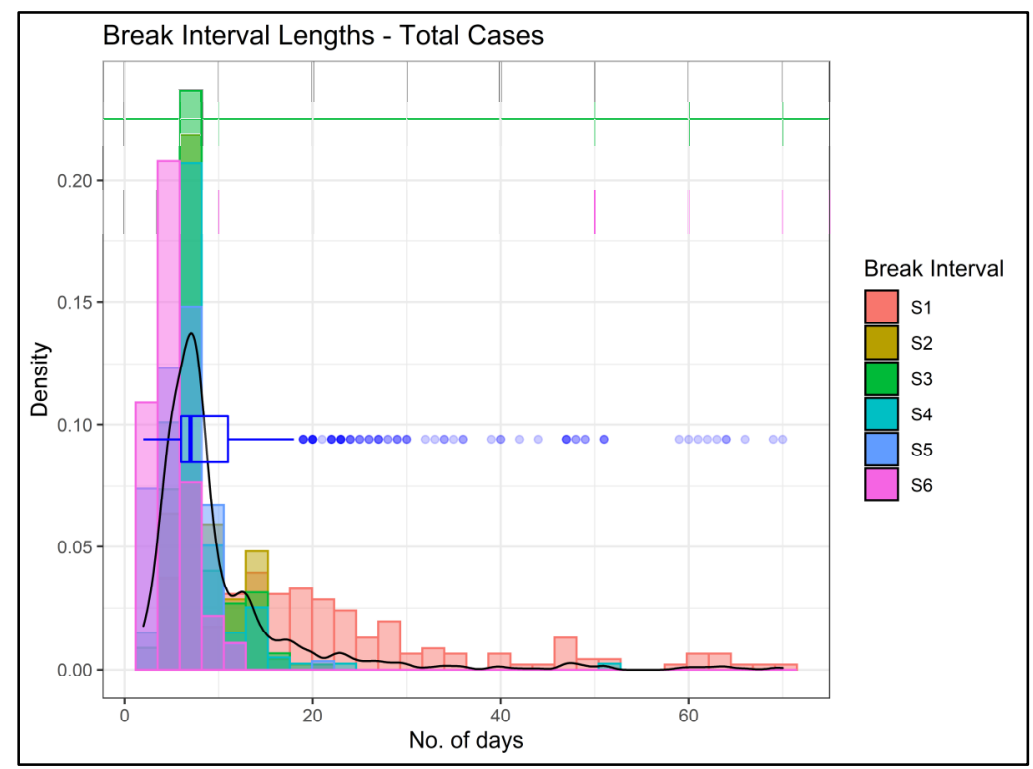

Figure 1: Density plot on overall break intervals

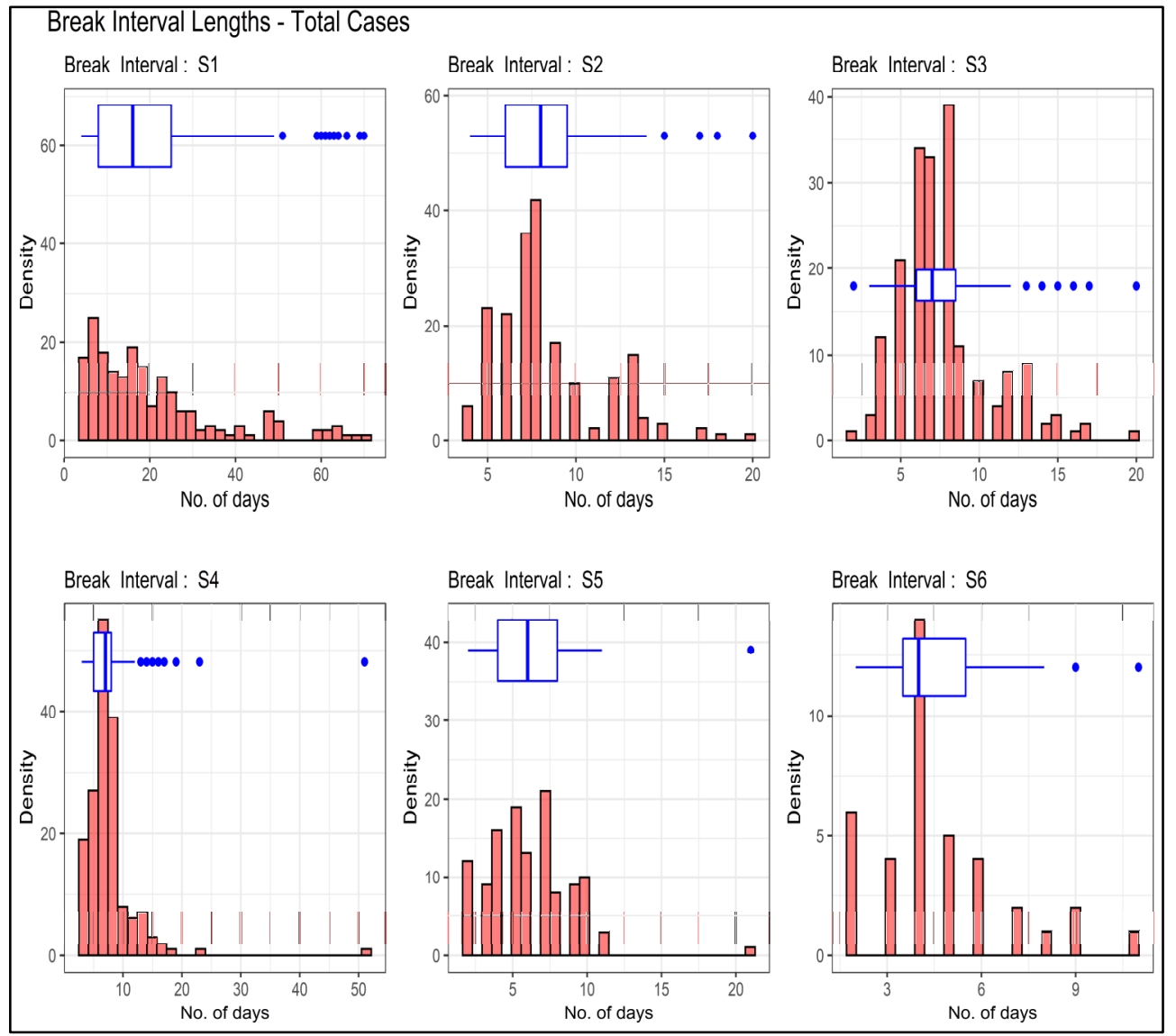

Figure 2: Histogram and box plot in each break interval 

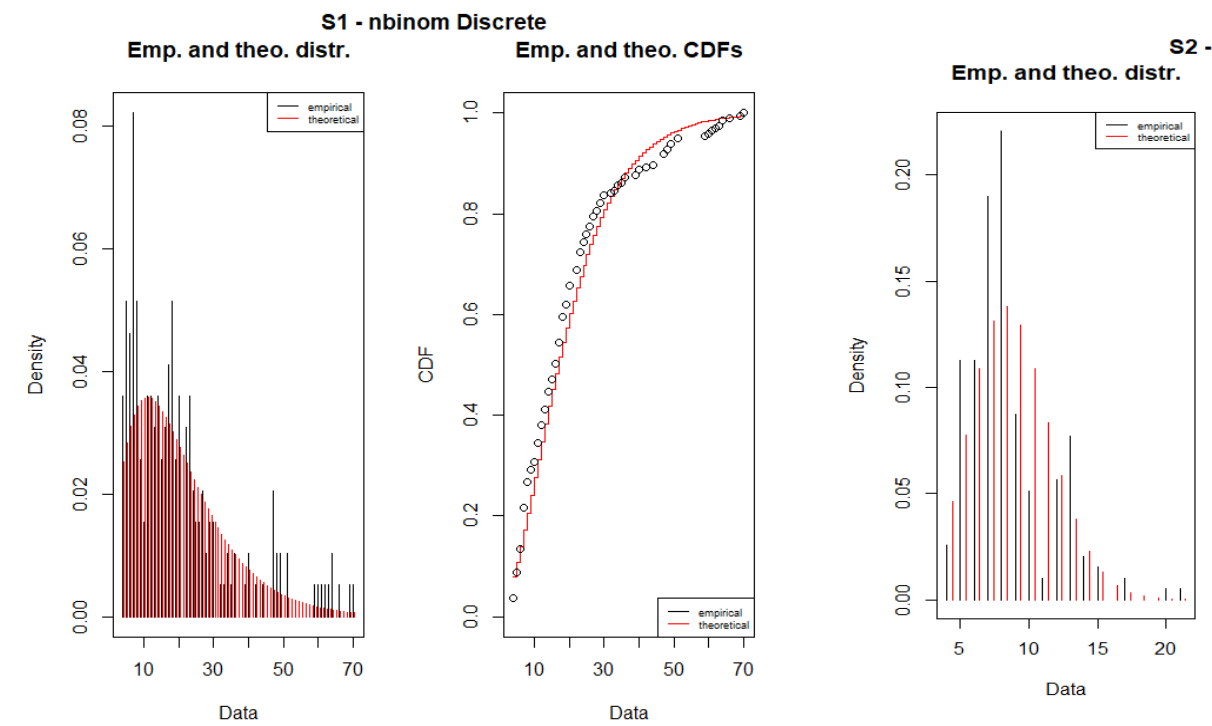

2 - pois Discrete

3 - nbinom Discrete

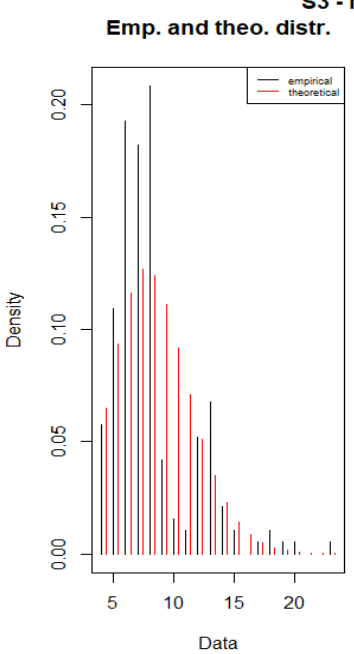

Emp. and theo. CDFs
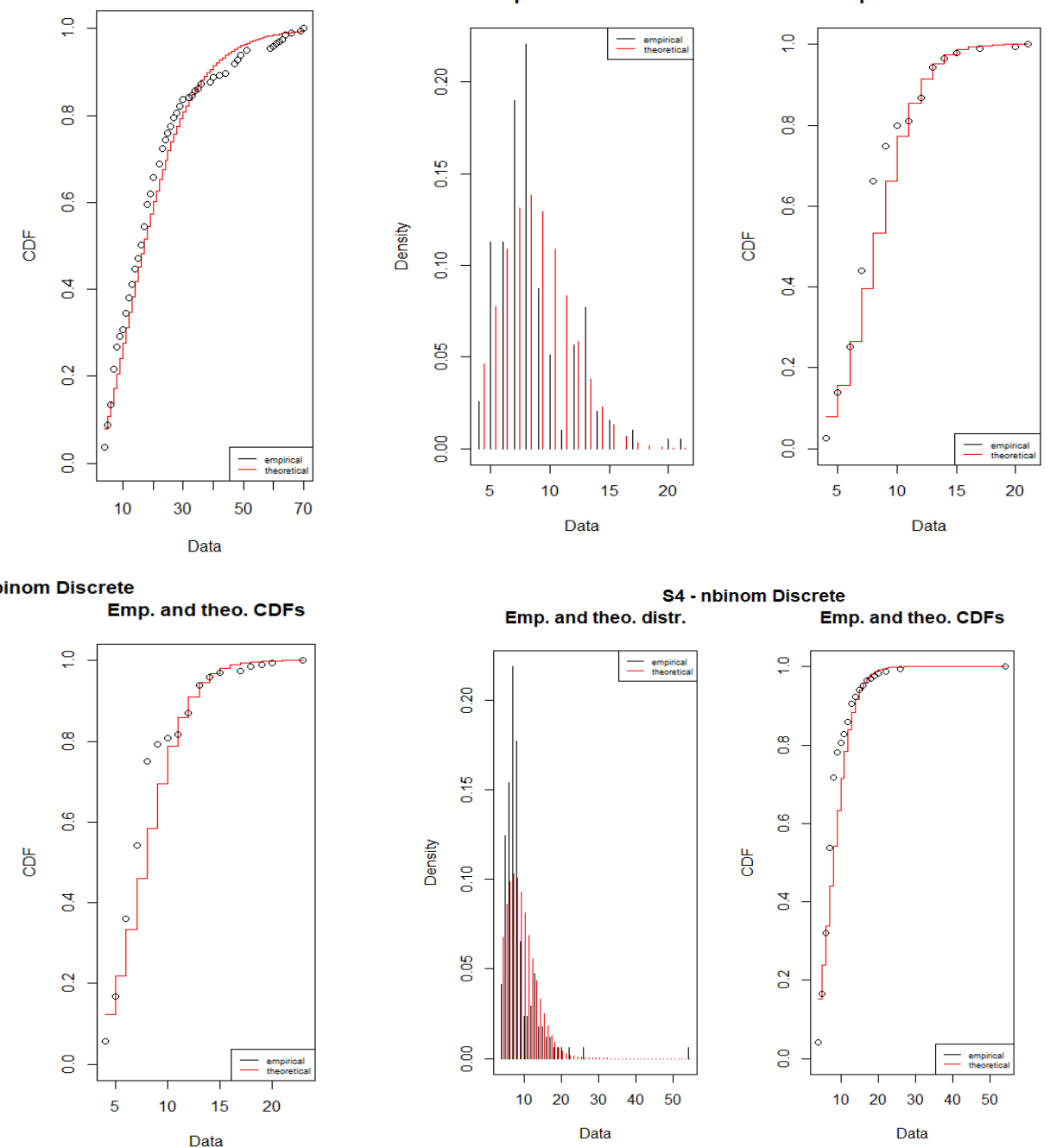$$
\text { binom Discrete }
$$

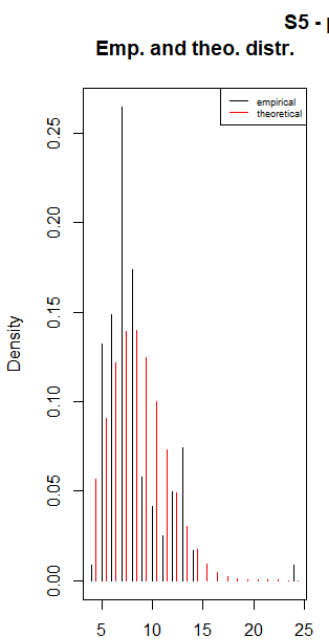

S5 - pois Discrete Emp. and theo. CDFs

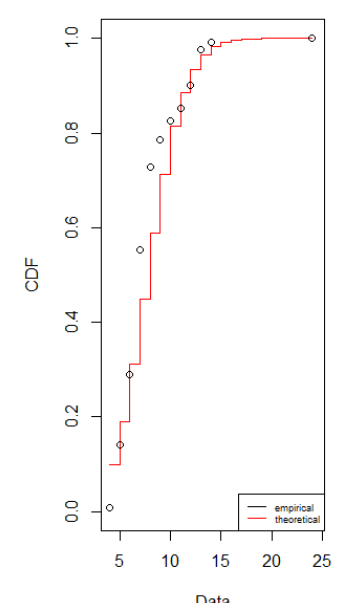

Emp. and theo. distr.
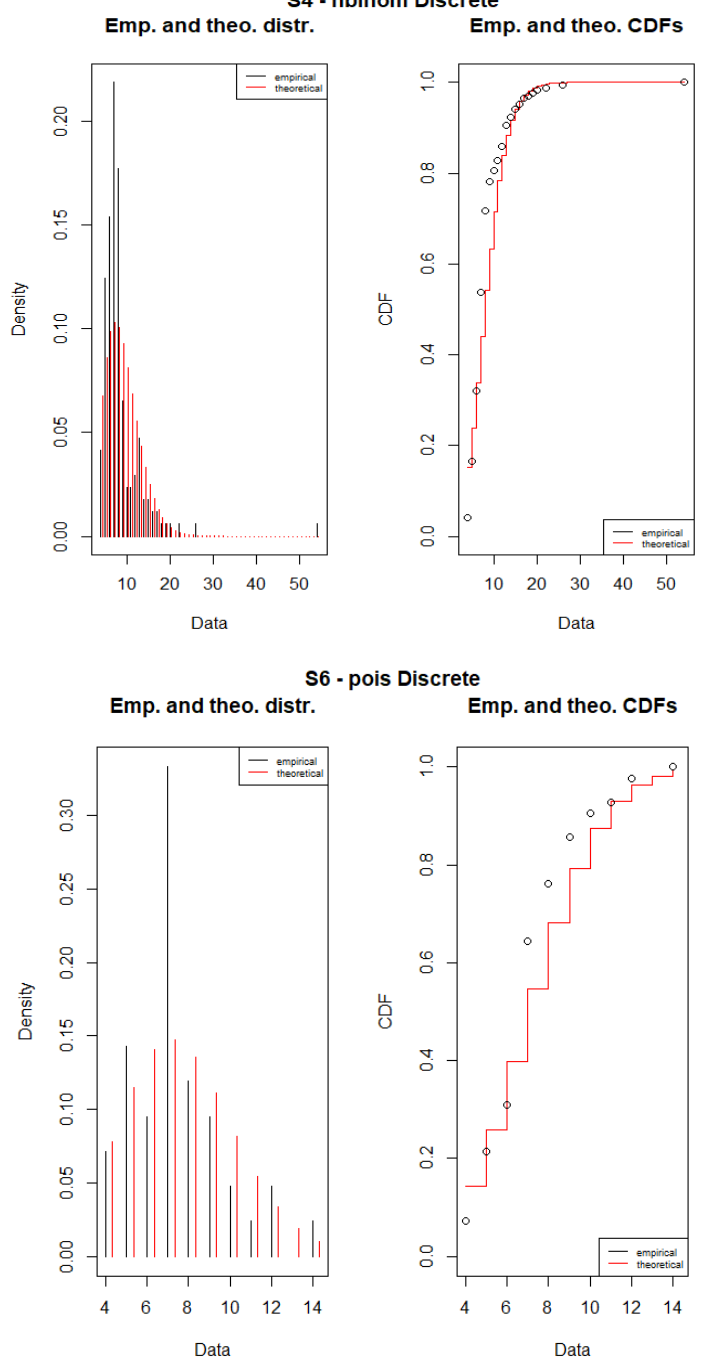

Data

Figure 3: Best fitted discrete distributions in Break Intervals 


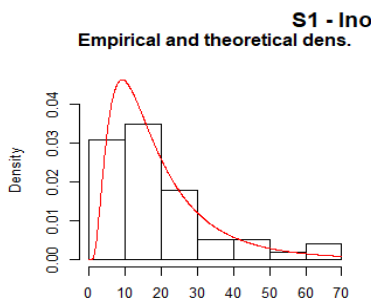

Data

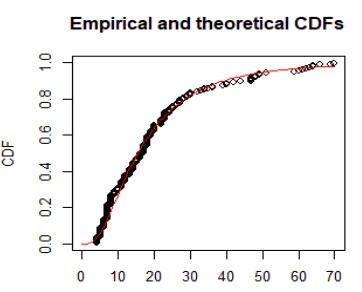

Data

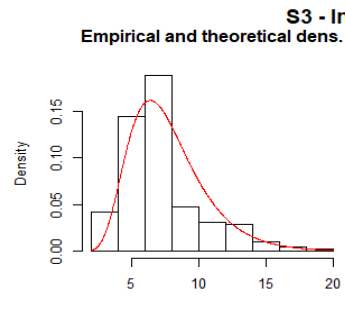

Data

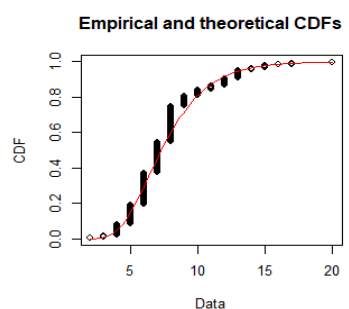

Data

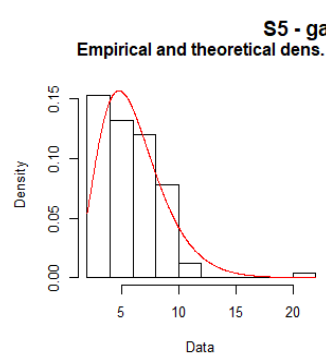

S5 - gamm
cal dens.
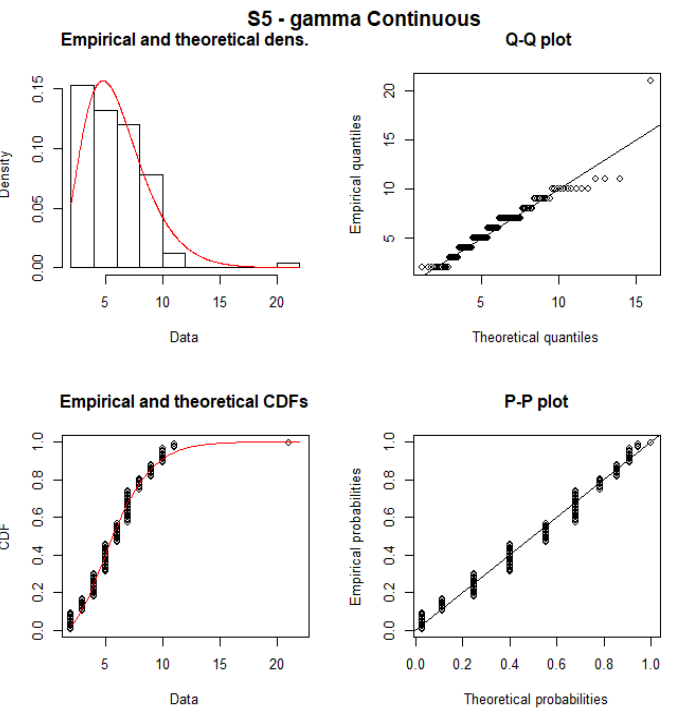
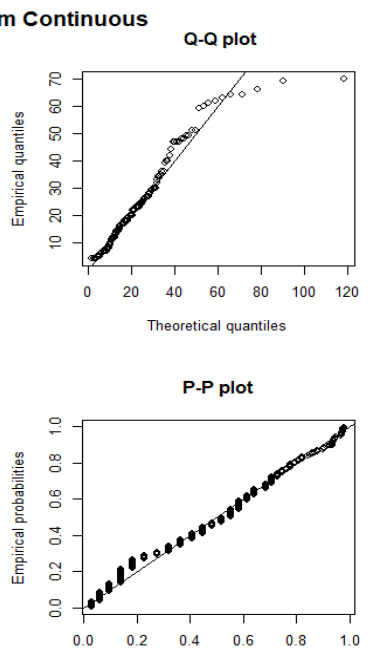

Theoretical probabilities
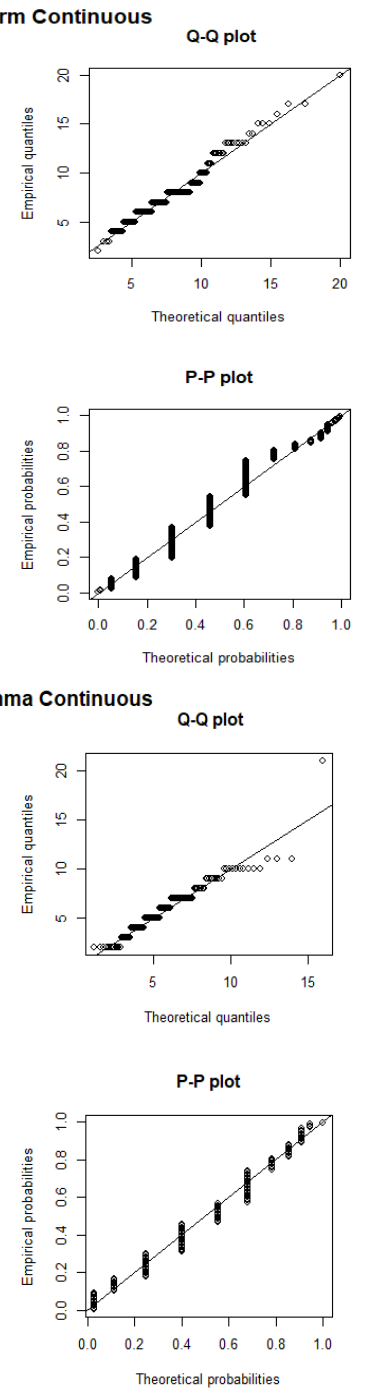

S2 - Inorm Continuous
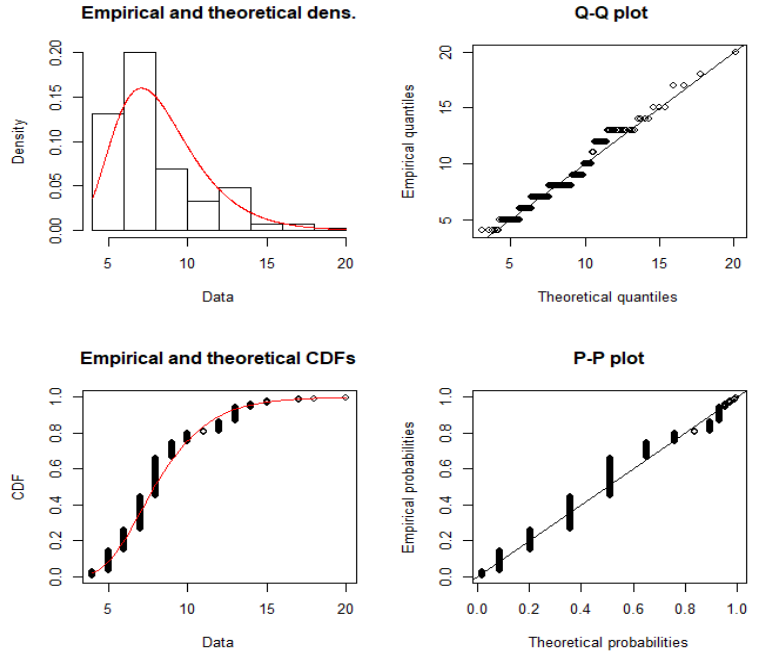

S4 - Inorm Continuous
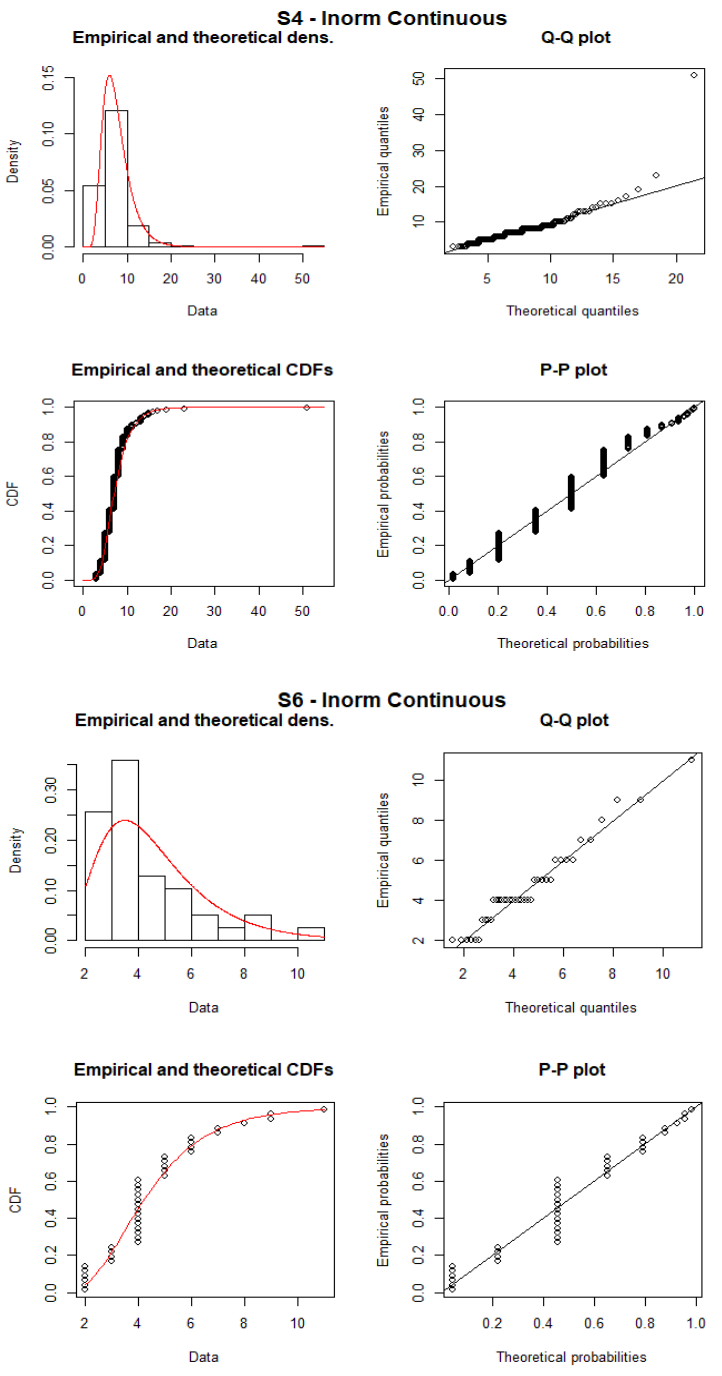

Figure 4: Best fitted continuous distributions in break intervals 


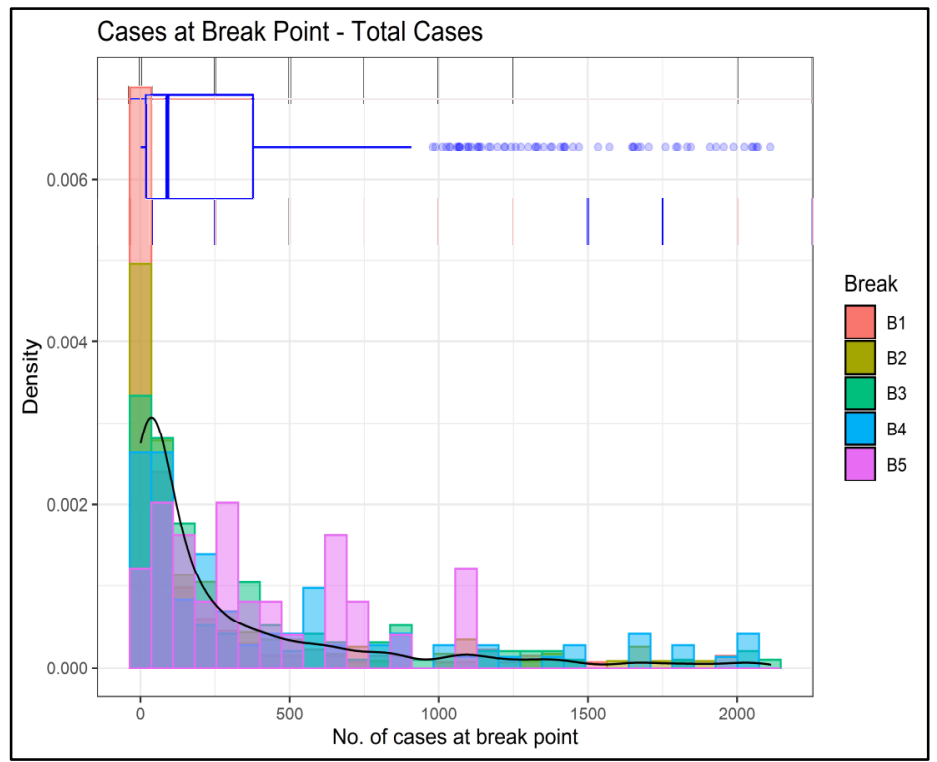

Figure 5: Density plot on overall break intervals
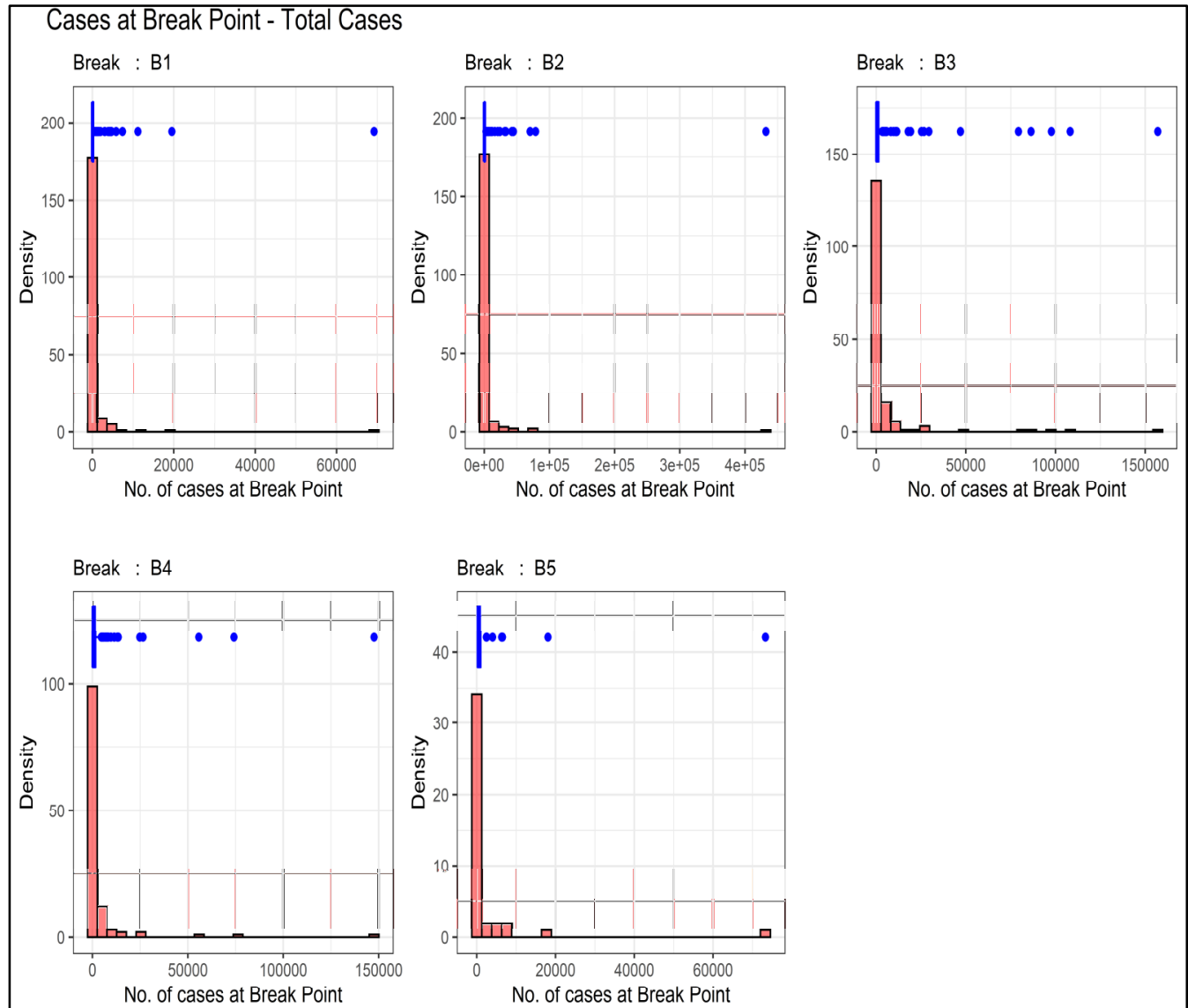

Figure 6: Histogram and box plot in each break interval 

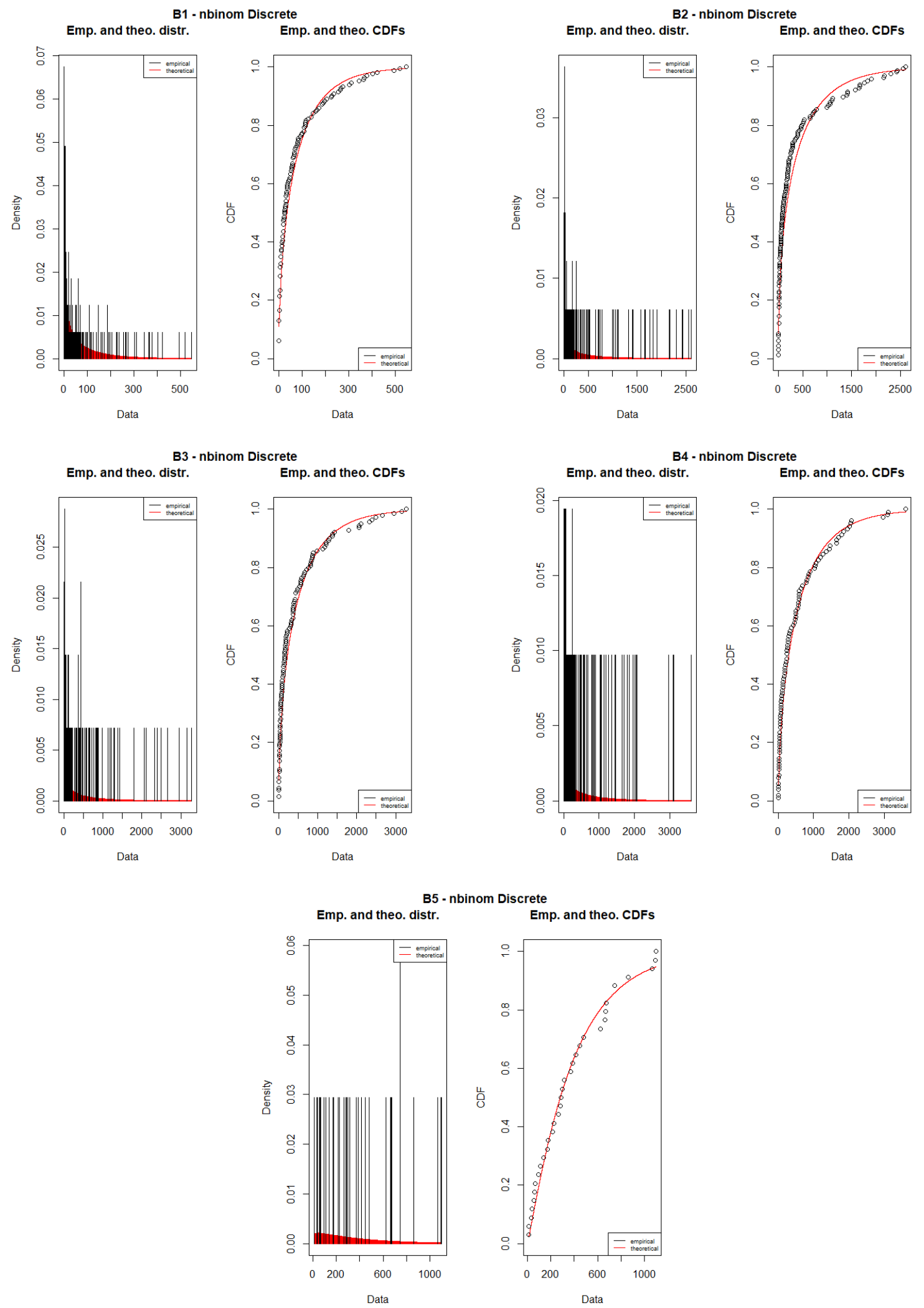

Figure 7: Best fitted discrete distribution of total cases at breakpoints 


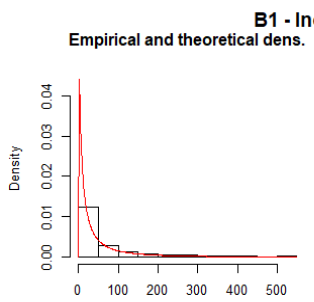

Data

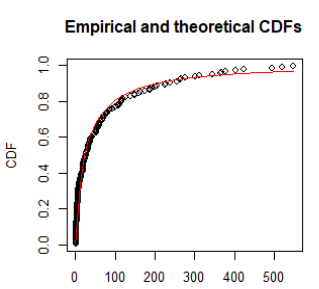

Data

B3 - Inorm Continuous

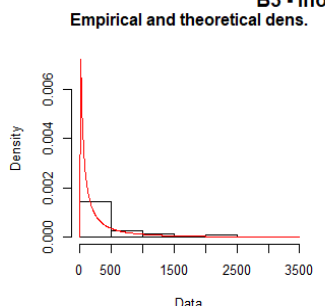

Data

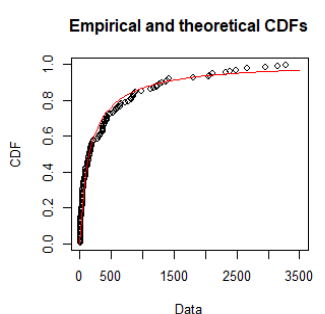

Data

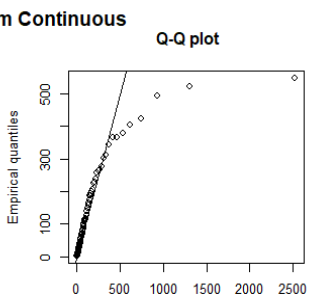

Theoretical quantiles

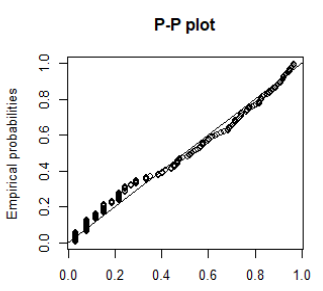

Theoretical probabilities
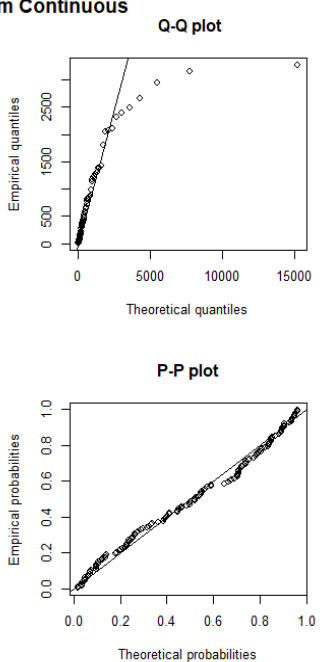

B5 -
-

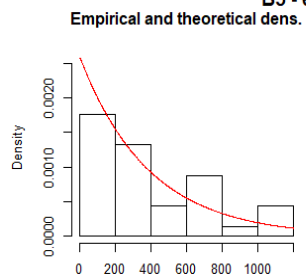

Data

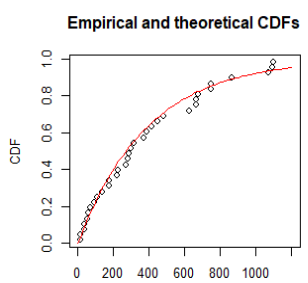

Data
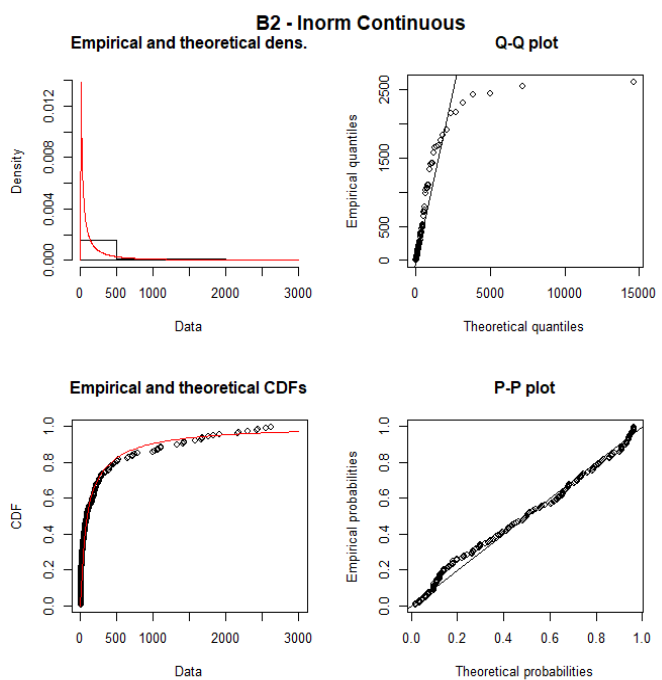

B4 - Inorm Continuous

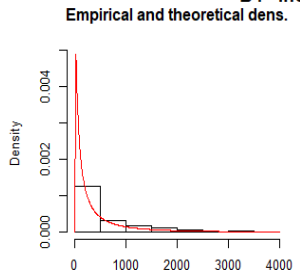

Data
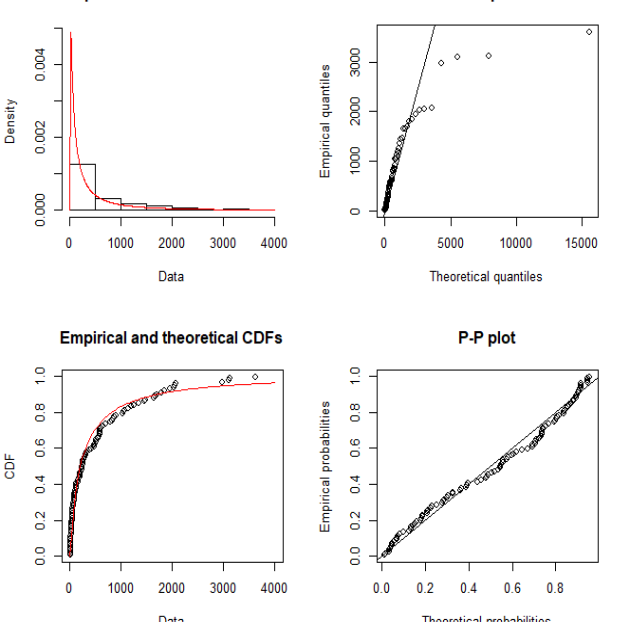

Data

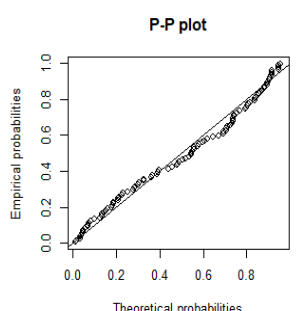

Continuous

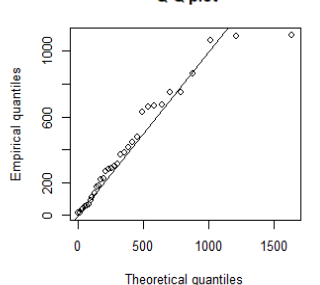

P-P plot

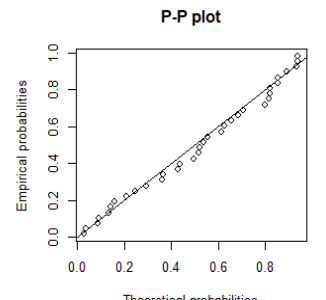

Figure 8: Best fitted continuous distributions of cases at breakpoints 
Table 1: Descriptive statistics of break interval based on number of days

\begin{tabular}{|c|c|c|c|c|c|c|}
\hline \multicolumn{7}{|c|}{ Table 1: Descriptive statistics of break interval based on number of days } \\
\hline Break Interval & Min. & $1^{\text {st }} \mathrm{Qu}$. & Median & Mean & $3^{\text {rd }} \mathrm{Qu}$. & Max. \\
\hline $\mathrm{S}_{1}$ & 4 & 8 & 16 & 20.04 & 25 & 70 \\
\hline $\mathrm{S}_{2}$ & 4 & 6.5 & 8 & 8.426 & 9.5 & 21 \\
\hline $\mathrm{S}_{3}$ & 4 & 6 & 7 & 8.073 & 8.25 & 23 \\
\hline $\mathrm{S}_{4}$ & 4 & 6 & 7 & 8.562 & 9 & 54 \\
\hline $\mathrm{S}_{5}$ & 4 & 6 & 7 & 8.025 & 9 & 24 \\
\hline $\mathrm{S}_{6}$ & 4 & 6 & 7 & 7.357 & 8 & 14 \\
\hline
\end{tabular}

\begin{tabular}{|c|c|c|c|c|}
\hline \multicolumn{5}{|c|}{ Table 2: Distribution fitting on Break Intervals } \\
\hline \multicolumn{2}{|r|}{ Distribution } & AIC & $\mathrm{BIC}$ & Interval \\
\hline Normal & mean $=20.041, \mathrm{sd}=15.193$ & 1618.504 & 1625.050 & \multirow{5}{*}{$\mathrm{S} 1$} \\
\hline NegBinom & size $=2.289, \mathrm{mu}=20.036$ & 1519.761 & 1526.307 & \\
\hline Poisson & lambda $=20.041$ & 2845.370 & 2848.643 & \\
\hline LogNormal & meanlog $=2.737$, sdlog $=0.728$ & 1500.993 & 1507.539 & \\
\hline Gamma & shape $=1.74$, rate $=0$ & 1517.354 & 1523.900 & \\
\hline Normal & mean $=8.426, \mathrm{sd}=3.004$ & 986.363 & 992.909 & \multirow{5}{*}{$\mathrm{S} 2$} \\
\hline NegBinom & size $=143.893, \mathrm{mu}=8.426$ & 963.021 & 969.567 & \\
\hline Poisson & lambda $=8.426$ & 961.411 & 964.684 & \\
\hline LogNormal & meanlog $=2.069$, sdlog $=0.334$ & 936.440 & 942.986 & \\
\hline Gamma & shape $=7.952$, rate $=1$ & 946.518 & 953.064 & \\
\hline Normal & mean $=8.073, \mathrm{sd}=3.294$ & 1006.612 & 1013.127 & \multirow{5}{*}{ S3 } \\
\hline NegBinom & size $=30.527, \mathrm{mu}=8.073$ & 969.217 & 975.732 & \\
\hline Poisson & lambda $=8.073$ & 974.439 & 977.696 & \\
\hline LogNormal & meanlog $=1.981$, sdlog $=0.364$ & 917.121 & 923.625 & \\
\hline Gamma & shape $=6.884$, rate $=1$ & 923.273 & 929.777 & \\
\hline Normal & mean $=8.562, \mathrm{sd}=5.031$ & 1029.682 & 1035.942 & \multirow{5}{*}{ S4 } \\
\hline NegBinom & size $=9.199, \mathrm{mu}=8.562$ & 925.052 & 931.312 & \\
\hline Poisson & lambda $=8.562$ & 999.098 & 1002.228 & \\
\hline LogNormal & meanlog $=1.946$, sdlog $=0.408$ & 838.067 & 844.326 & \\
\hline Gamma & shape $=2.865$, rate $=0$ & 897.692 & 903.951 & \\
\hline Normal & mean $=8.025, \mathrm{sd}=2.827$ & 598.861 & 604.453 & \multirow{5}{*}{ S5 } \\
\hline NegBinom & size $=182946.75, \mathrm{mu}=8.025$ & 580.531 & 586.123 & \\
\hline Poisson & lambda $=8.025$ & 578.531 & 581.327 & \\
\hline LogNormal & meanlog $=1.693$, sdlog $=0.488$ & 583.292 & 588.883 & \\
\hline Gamma & shape $=4.689$, rate $=1$ & 578.328 & 583.919 & \\
\hline Normal & mean $=7.357, \mathrm{sd}=2.202$ & 189.496 & 192.971 & \multirow{5}{*}{ S6 } \\
\hline NegBinom & size $=2332625.794, \mathrm{mu}=7.357$ & 190.746 & 194.221 & \\
\hline Poisson & lambda $=7.357$ & 188.746 & 190.484 & \\
\hline LogNormal & meanlog $=1.434$, sdlog $=0.438$ & 162.131 & 165.458 & \\
\hline Gamma & shape $=4.968$, rate $=1$ & 163.179 & 166.506 & \\
\hline
\end{tabular}




\begin{tabular}{|c|c|c|c|c|}
\hline Break & $\begin{array}{c}\text { No. of } \\
\text { countries }\end{array}$ & $\begin{array}{l}\text { No. of } \\
\text { outliers }\end{array}$ & $\begin{array}{c}\text { Outlier } \\
(\%)\end{array}$ & Country-Cases \\
\hline B1 & 195 & 32 & $16 \%$ & $\begin{array}{l}\text { Austria - 1332, Belarus - 700, Belgium - 1243, Brazil - 4256, } \\
\text { Canada - 4675, Chile - 1142, China - 1988, Ecuador - 789, } \\
\text { France - 4499, Germany - 3062, India - 1071, Indonesia - 579, } \\
\text { Iran - 5823, Ireland - 785, Israel - 1930, Italy - 3858, Japan - } \\
\text { 1128, Mexico - 848, Netherlands - 2994, Peru - 1323, Poland - } \\
\text { 901, Portugal - 642, Qatar - 634, Russia - 1534, Saudi Arabia - } \\
\text { 1012, Singapore - 594, Spain - 11178, Sweden - 1167, Turkey } \\
\text { - 7402, Switzerland - 3010, United Kingdom - 19522, United } \\
\text { States - } 69194\end{array}$ \\
\hline B2 & 192 & 27 & $14 \%$ & $\begin{array}{l}\text { Austria - 5888, Belarus - 3281, Belgium - 10836, , Canada - } \\
\text { 20748, Chile - 3031, China - 44724, , Germany - 42288, India } \\
\text { - 7447, Iran - 16169, , Italy - 31506, Japan - 3906, Netherlands } \\
\text { - 9762, , Portugal - 2995, Russia - 11917, Saudi Arabia - 2932, } \\
\text {, Spain - 78797, Sweden - 3700, Switzerland - 10714, , United } \\
\text { Arab Emirates - 2990, United Kingdom - 70272, United States } \\
\text { - } 432132\end{array}$ \\
\hline B3 & 169 & 30 & $18 \%$ & $\begin{array}{l}\text { Australia - 5844, Austria - 11129, Belgium - 26667, Chile - } \\
\text { 5546, China - 79355, Czech Republic - 3589, Ecuador - 4965, } \\
\text { Germany - 108202, Iran - 29406, Ireland - 5364, Israel - } \\
\text { 10743, Malaysia - 4228, Mexico - 5399, Netherlands - 17851, } \\
\text { Peru - 11475, Poland - 5205, Portugal - 8251, Qatar - 3428, } \\
\text { Romania - 4057, South Korea - 8162, Spain - 157022, Sweden } \\
\text { - 9685, Switzerland - 19227, Ukraine - } 4161\end{array}$ \\
\hline B4 & 121 & 18 & $15 \%$ & $\begin{array}{l}\text { Austria - 13560, Chile - 8273, Czech Republic - 5312, } \\
\text { Indonesia - 5136, Iran - 55743, Ireland - 11479, Italy - 147577, } \\
\text { Norway - 5208, Pakistan - 5988, Poland - 7582, Portugal - } \\
\text { 13141, Serbia - 4873, South Korea - 9661, Switzerland - } \\
\text { 24820, Turkey - 74193 }\end{array}$ \\
\hline B5 & 42 & 8 & $19 \%$ & $\begin{array}{l}\text { Argentina - 2432, Czech Republic - 6303, Denmark - 6511, } \\
\text { Iran - 73303, Norway - 6415, Panama - 4016, Portugal - } \\
\text { 18091, South Africa - } 2506\end{array}$ \\
\hline
\end{tabular}




\begin{tabular}{|c|c|c|c|c|c|c|}
\hline \multicolumn{6}{|c|}{ Table 4: Descriptive statistics of break interval based on number of cases } \\
\hline Break & Min. & $1^{\text {st }}$ Qu. & Median & Mean & $3^{\text {rd }}$ Qu. & Max. \\
\hline $\mathrm{B}_{1}$ & 1 & 6 & 26 & 74.398 & 84.5 & 549 \\
\hline $\mathrm{B}_{2}$ & 2 & 18 & 87 & 364.115 & 339 & 2615 \\
\hline $\mathrm{B}_{3}$ & 4 & 39.5 & 158 & 469.136 & 571.5 & 3277 \\
\hline $\mathrm{B}_{4}$ & 5 & 51.5 & 241 & 579.805 & 802 & 3614 \\
\hline $\mathrm{B}_{5}$ & 12 & 117.2 & 293.5 & 388.500 & 653 & 1100 \\
\hline
\end{tabular}

\begin{tabular}{|c|c|c|c|c|}
\hline \multicolumn{5}{|c|}{ Table 5: Distribution fitting on number of cases at breaks } \\
\hline & Distribution & AIC & BIC & Break \\
\hline Exponential & rate $=0.013$ & 1732.877 & 1735.971 & \\
\hline LogNormal & meanlog $=3.133$, sdlog $=1.716$ & 1664.021 & 1670.208 & \\
\hline Gamma & shape $=0.446$, rate $=0$ & 1682.891 & 1689.078 & B1 \\
\hline Normal & mean $=74.399, \mathrm{sd}=111.387$ & 2003.014 & 2009.202 & \\
\hline NegBinom & size $=0.535, \mathrm{mu}=74.419$ & 1686.026 & 1692.213 & \\
\hline Poisson & lambda $=74.399$ & 20783.846 & 20786.94 & \\
\hline Exponential & rate $=0.003$ & 2278.165 & 2281.271 & \\
\hline LogNormal & meanlog $=4.473, \mathrm{sdlog}=1.866$ & 2154.149 & 2160.361 & \\
\hline Gamma & shape $=0.362$, rate $=0$ & 2187.982 & 2194.194 & B2 \\
\hline Normal & mean $=364.115, \mathrm{sd}=605.353$ & 2586.168 & 2592.38 & \\
\hline NegBinom & size $=0.452, \mathrm{mu}=364.262$ & 2184.135 & 2190.347 & \\
\hline Poisson & lambda $=364.115$ & 118204.847 & 118207.953 & \\
\hline Exponential & rate $=0.002$ & 1989.949 & 1992.883 & \\
\hline LogNormal & meanlog $=4.967$, sdlog $=1.735$ & 1932.466 & 1938.335 & \\
\hline Gamma & shape $=0.451$, rate $=0$ & 1946.092 & 1951.961 & B3 \\
\hline Normal & mean $=469.137, \mathrm{sd}=698.406$ & 2219.032 & 2224.9 & \\
\hline NegBinom & size $=0.531, \mathrm{mu}=469.156$ & 1944.489 & 1950.358 & \\
\hline Poisson & lambda $=469.137$ & 107695.872 & 107698.807 & \\
\hline Exponential & rate $=0.002$ & 1518.715 & 1521.35 & \\
\hline LogNormal & meanlog $=5.296$, sdlog $=1.685$ & 1494.836 & 1500.105 & \\
\hline Gamma & shape $=0.566$, rate $=0$ & 1495.598 & 1500.868 & B4 \\
\hline Normal & mean $=579.806, \mathrm{sd}=770.847$ & 1665.684 & 1670.954 & \\
\hline NegBinom & size $=0.582, \mathrm{mu}=579.74$ & 1496.071 & 1501.34 & \\
\hline Poisson & lambda $=579.806$ & 85921.07 & 85923.705 & \\
\hline Exponential & rate $=0.003$ & 475.436 & 476.962 & \\
\hline LogNormal & meanlog $=5.452$, sdlog $=1.197$ & 483.439 & 486.492 & \\
\hline Gamma & shape $=1.457$,rate $=0$ & 478.832 & 481.885 & B5 \\
\hline Normal & mean $=388.5, \mathrm{sd}=321.866$ & 493.129 & 496.182 & \\
\hline NegBinom & size $=1.123, \mathrm{mu}=388.62$ & 477.247 & 480.3 & \\
\hline Poisson & lambda $=388.5$ & 9466.545 & 9468.072 & \\
\hline
\end{tabular}

